\title{
Insight of Direct Search Methods and Module-Integrated Algorithms for Maximum Power Point Tracking (MPPT) of Stand-Alone Photovoltaic Systems
}

\author{
Jieming Ma ${ }^{1,2, *}$, Ka Lok Man ${ }^{1,3}$, T.O. Ting ${ }^{1}$, Hyunshin Lee ${ }^{3}$, Taikyeong Jeong ${ }^{3}$, \\ Jong-Kug Sean ${ }^{4}$, Sheng-Uei Guan ${ }^{1}$, and Prudence W.H. Wong ${ }^{2}$ \\ ${ }^{1} \mathrm{Xi}$ ' an Jiaotong, Liverpool University, China \\ ${ }^{2}$ University of Liverpool, UK \\ ${ }^{3}$ Myongji University, South Korea \\ ${ }^{4}$ LS Industrial Systems, South Korea \\ jieming@liv.ac.uk
}

\begin{abstract}
By detection of input signal, Maximum Power Point Tracking (MPPT) algorithms are used to maximize the potential output power. Since Perturbation and Observe (P\&O) method was first applied in a Photovoltaic (PV) system, a myriad of MPPT algorithms has been proposed. With the pros and cons of various MPPT algorithms, a detailed analysis of several typical direct search and module-integrated MPPT algorithms is presented in this paper. Directions of future work for implementing new MPPT algorithms are also outlined.
\end{abstract}

Keywords: Maximum Power Point Tracking (MPPT) algorithms, Photovoltaic (PV) system, Perturbation and observe (P\&O) method.

\section{Introduction}

To address the environmental issue, Photovoltaic (PV) solar generation has attracted increasing attention as one of the most potential and promising alternative energy sources. By taking the advantage of directly converting solar energy into current electricity, PV stands as a paradigm of reducing carbon dioxide emission, and the use of PV has been proposed by many countries.

Recent research has shown that the performance of PV modules is sensitive to the operating environment [9], and therefore the output Current-Voltage (I-V) characteristic curve of commercial PV modules exhibited nonlinear characteristics. Fig. 1 shows the variation of the I-V curves under different irradiation and temperature conditions. It can be observed that the Maximum Power Point (MPP) [3], at which the PV generator delivers the maximum output power, varies with these weather conditions.

In order to gain maximum energy conversion efficiency, researchers have proposed a series of novel algorithms for tracking the MPPs. In terms of the control strategies, Maximum Power Point Tracking (MPPT) algorithms can be classified as direct search methods and module-integrated algorithms. Direct search methods, which

\footnotetext{
* Corresponding author.
} 
profit from its simple implementation, have been most commonly used in practice for a long time. Based on the measurements of PV electricity, direct seeking methods locate the operating point without considering any environment factors. Moduleintegrated algorithms, however, usually require a large number of experimental data or mathematical functions deduced from the physical properties of PV materials. In most cases, thermometers and light meters are applied in module-integrated PV systems to evaluate the atmospheric factors. Not only the additional measurements increase the hardware cost, but their accuracy also affects the performance of MPPT algorithms. Thus, traditional module-integrated approaches were thought to be expensive and unreliable [14]. That is the most likely reason why one particular direct MPPT algorithm (e.g. Perturb-and-Observe (P\&O) [8]) with imperfect tracking performance claimed by many researchers, continuously has exuberant vitality.

With recent advances in the field of Integrated Circuit (IC) manufacturing technology, the performance of hardware toolkits has been improved tremendously while the cost has dropped significantly. All these aspects have led to a renewed interest in module-integrated MPPT algorithms. Many module-integrated microcontroller-based PV systems have shown flexibility and reliable performance in MPPT compared with conventional direct approaches [4].

The advantages and disadvantages of direct search methods for MPPT are discussed in the next section. Section 3 illustrates two practical module-integrated algorithms: Particle Swarm Optimization (PSO [4]) and Golden Section Search (GSS [15]). The last section is devoted to discussions and conclusions along with directions for future work.

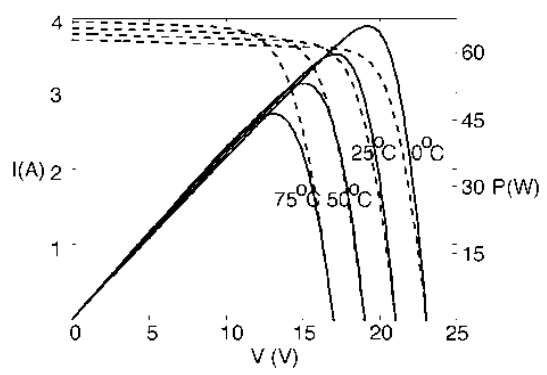

(a)

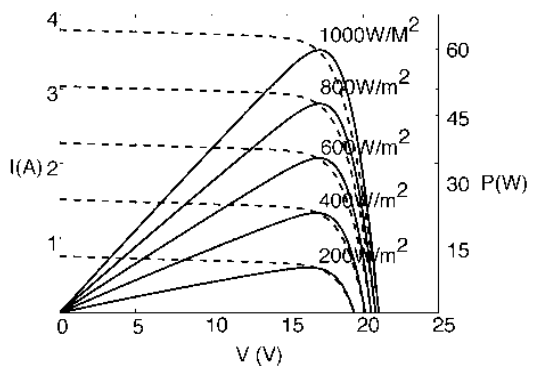

(b)

Fig. 1. The variation of the P-V and I-V curves under (a) varying temperature and (b) varying irradiance

\section{Direct Search MPPT Methods}

Since Hill Climbing algorithms appeared in literature on power electronics in 80's of the last century, many new direct MPPT methods have emerged in a rapid succession $[8,2,13,7]$. The common advantage of the cohort of algorithms is their environmentindependent features. Environmental measurements and sampling technologies have not been widely used over the last two decades due to their immaturity and faultiness. A small quantity of measurements not only means the lower cost that can be attained, but also indicates the higher accuracy and reliability that can be achieved. 


\subsection{Perturbation and Observe $(\mathbf{P} \& O)$ Method}

$\mathrm{P} \& \mathrm{O}$ is a well-known practical MPPT algorithm and is presented in the literature as a reference method. On the basis of the Power-Voltage (P-V) characteristics curve of a $\mathrm{PV}$ module, $\mathrm{P} \& \mathrm{O}$ method perturbs the operating point and determines the change of direction by comparing the power with the historical reference value.

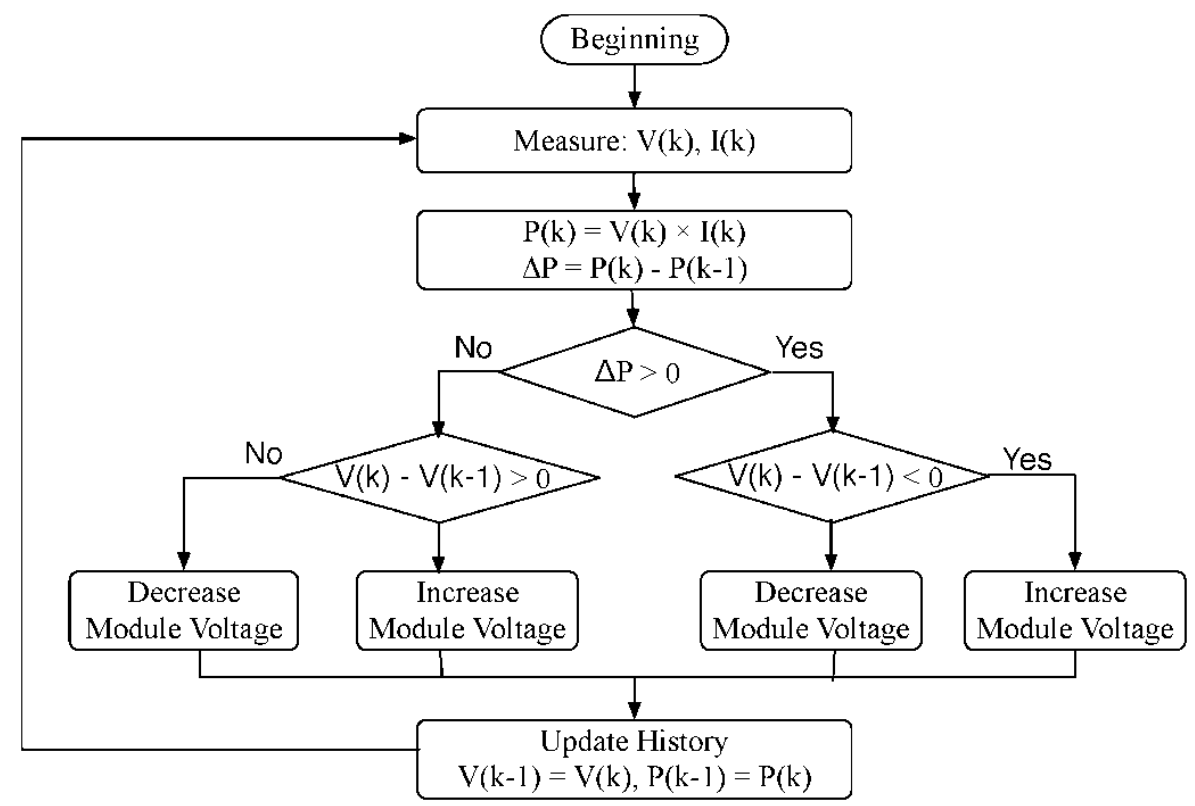

Fig. 2. Conventional Perturbation and Observe algorithm flowchart

Fig. 2 shows a flowchart for the basic algorithm for the most basic form. Starting from the measures of the operating voltage and current, the algorithm firstly obtains the sign of $\Delta \mathrm{P}$, which denotes the difference between the current operating power and the reference power. When the sign of $\Delta \mathrm{P}$ is positive, the direction of assigned increment is identical to that of the perturbation of the operating voltage. On the contrary, it is opposite as long as the $\Delta \mathrm{P}$ is negative.

A P\&O algorithm is independent of PV generator characteristics and thus its simplicity and flexibility are widely recognized by many the researchers. However, the shortcomings of $\mathrm{P} \& \mathrm{O}$ have been proven in numerous papers since $\mathrm{P} \& \mathrm{O}$ was firstly applied in PV systems. Even in an ideal environment, the operating point oscillates around the MPP, which significantly affects the tracking efficiency. The easiest way of improving the oscillation is to apply smaller increments. However, this approach will slow down the tracking speed. A clever variable perturbation step size is introduced in [2]. The flexible increment can avoid intense oscillation while at the same time keeping the acceptable efficiency from the start of tracking stage. The minor disadvantage comes from the approximation module based framework, which increases the complexity of the algorithm. The accuracy of the module, like many used in module-integrated methods, directly affects the seeking efficiency. 
The weather conditions, which are transformers of $\mathrm{P}-\mathrm{V}$ characteristic, are hard to predict and are usually non-linear. Since $\mathrm{P} \& \mathrm{O}$ algorithm refers to a particular hill-like $\mathrm{P}-\mathrm{V}$ curve monotonously, the weakness of tracking changing MPP is obvious. As has been illustrated in [8], P\&O method fails under rapidly changing atmospheric conditions. The seeking direction may be set wrong as long as the irradiance and/or temperature change(s) within a sampling time. In addition, the P-V characteristic curve will show multi-peak property under partial shadows [11], in which P\&O MPPT technique may fail in tracking the global MPP.

\subsection{Incremental Conductance (IncCond) Method}

IncCond method was implemented to overcome the limitations of P\&O MPPT algorithms under a rapidly changing environment and was firstly introduced in [8]. By comparing the incremental and instantaneous conductance of PV modules, the control process can detect the variety of atmospheric conditions (e.g. solar radiation and temperature) and correct the failure situation of the conventional $\mathrm{P} \& \mathrm{O}$ algorithm.

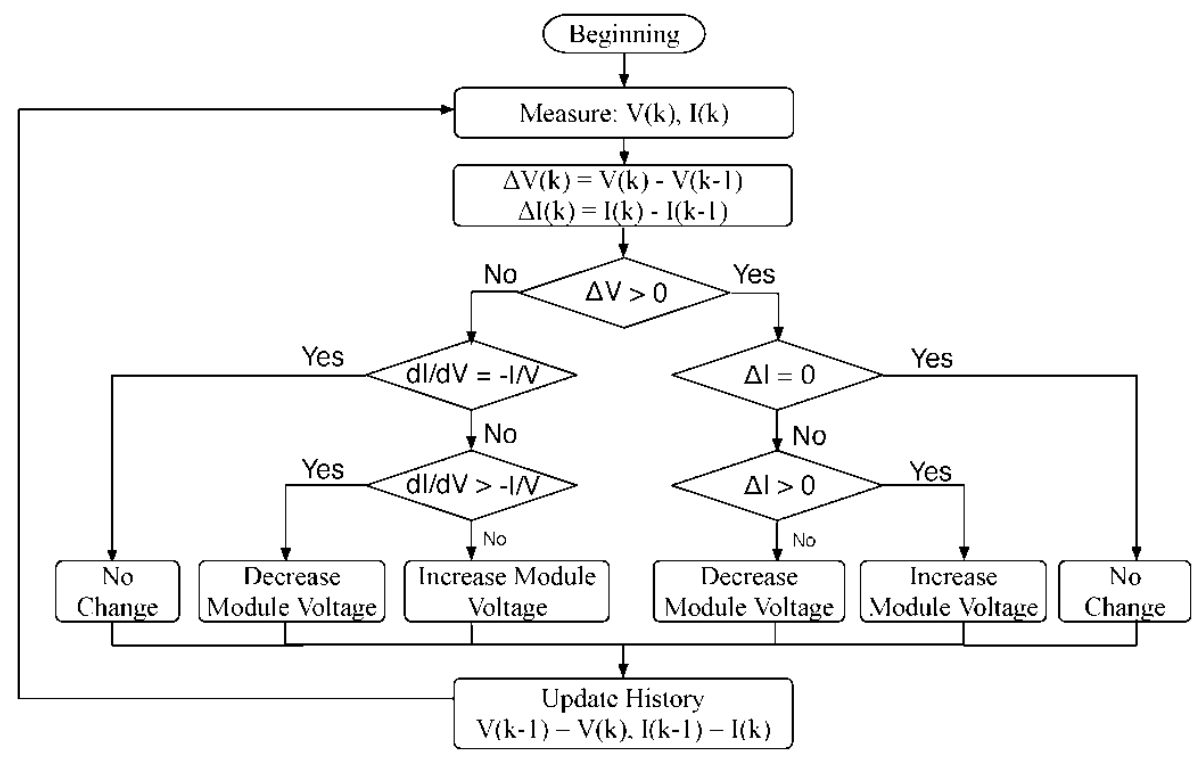

Fig. 3. Conventional Incremental Conductance algorithm flowchart

An algorithm flowchart of the IncCond method is shown in Fig. 3. The IncCond algorithm starts from detecting the changes of weather conditions. The increase of the irradiation can be recognized by $\mathrm{dV}=0$ and $\mathrm{dI}>0$, while $\mathrm{dV}=0$ and $\mathrm{dI}<0$ can determine the decrease of the irradiation. The increment voltage is given as the direction of the irradiation. If changes in voltage and current are both detected, the control of the operating voltage will be based on the relationship between the location of operating point in $\mathrm{P}-\mathrm{V}$ curve and the sign of module conductance. When the operating point is located at the left side of MPP, the sign of the conductance is negative. On the contrary, the conductance 
turns out to be positive as long as the operating point is at the right-hand side of MPP. The value of the conductance drops to zero when it reaches the MPP. Since

$$
\frac{d P}{d V}=\frac{d(V \cdot I)}{d V}=I+V \frac{d I}{d V}
$$

these relationships can be given by

$$
\begin{aligned}
& \frac{\Delta P}{\Delta V}=-\frac{I}{V}, \text { at MPP } \\
& \frac{\Delta P}{\Delta V}>-\frac{I}{V}, \text { left of MPP } \\
& \frac{\Delta P}{\Delta V}<-\frac{I}{V}, \text { right of MPP }
\end{aligned}
$$

Though IncCond method fills in the blank of environment consideration, many of the inherent drawbacks, like oscillation and disability of particle shadow conditions, limit its application.

\section{Module-Integrated MPPT Algorithms}

Direct method is able to drive the operating voltage near MPP gradually, yet it usually requires much sampling time to obtain a satisfactory accuracy. Moreover, the characteristic of PV modules is shown nonlinear and related to many weather factors. It is difficult to determine the search direction in terms of the current and voltage only. Module-integrated MPPT algorithms are implemented based on the mathematical functions obtained from empirical data and can calculate the MPP directly. [4] also showed the flexibility and low cost of module-integrated methods. However, the conventional module-integrated MPPT algorithms were thought to be unfeasible since the performance is dependent on the accuracy of sensors and approximation modules. The complexity of the algorithm is significantly increased due to the calculation of the electrical property of the PV module (e.g. current, voltage or power). Even in a simple MPPT algorithm, more sampling time is required as long as the number of power point evaluation is large. It is well-known that the weather condition changes quickly at some times. If the sampling time is long, the operating voltage, which was estimated for the condition of the previous sample time, may be not proper for the current environment. The tracking efficiency is therefore limited by the lag of the control. Recent advances on PV module modeling and sensing technologies have led a renewable interest in module-integrated method. Two optimization algorithms were applied in [4] and [15], which describe the application of Particle Swarm Optimization and Golden Section Search in PV system respectively. The simulation results proved the MPPT efficiency of such algorithms is superior to that of direct search approaches.

\subsection{Particle Swarm Optimization (PSO)}

Particle Swarm Optimization (PSO) [10] is one of the prominent algorithms in the category of nature-inspired algorithms and it has been one of the most successful numerical 
optimization algorithms applied in many fields. One of the advantageous features of PSO is due to its ability to converge quickly to a potential solution. In other words, PSO is faster compared to many evolutionary algorithms such as Genetic Algorithm (GA) [5].

PSO works as follows. Firstly, candidate solutions (or commonly known as particles) are initially seeded onto the search space in a random manner. These particles will then move through the problem space with the aim of finding the global optimum. The movement is guided by the essentially important ingredient formulas:

$$
X_{i}^{t+1}=X_{i}^{t}+w V_{i}^{t-1}+2 r_{1}\left(X_{i}^{t}-\text { Pbest }_{i}^{t}\right)+2 r_{2}\left(X_{i}^{t}-\text { Gbest }_{i}^{t}\right)
$$

whereby $V_{i}^{t}$ is the velocity for $i^{t h}$ dimension at time $\mathrm{t}, \mathrm{w}$ is the inertia weight, usually is set to $0.5, X_{i}^{t}$ is the current position of $i^{t h}$ dimension at time t, Pbest $t_{i}^{t}$ is the best position for $i^{\text {th }}$ dimension at time $t$ of a particle, also known as personal best, Pbest $t_{i}^{t}$ is the best solution among all participating particles for $i^{\text {th }}$ dimension at time $\mathrm{t}$, also known as global best, $r_{1}$ and $r_{1}$ are independent uniform random numbers within $[0,1]$.

In each iteration, all particles will be evaluated through a similar cost function. Then, update of Pbest and Gbest values are performed instantly. In other words, the asynchronous update is adopted here. The reason for asynchronous update is that the information of Pbest and Gbest can be feedback into the whole population instantly without delay and this will accelerate the convergence rate.

During the update of velocity through [10], the limit of Vmax and Vmin is imposed, usually within $10 \%, 50 \%$ or $100 \%$ of search space. The value chosen for Vmax and Vmin is not really crucial and does not affect the performance drastically. Also, after the update through (2), checking is done to ensure that particles only explore the predefined search space. Many techniques are used to handle this boundary values. Simply set the value to boundary limit is one of the alternatives. Another alternative will be to impose re-initialization within the search space upon violation. The later alternative is preferred as this will increase the diversity of the population and hence assist in avoiding local optima. The similar boundary handling technique is adopted in this work. As the number of iteration increases, particles accelerate towards those with better fitness until maximum iteration is reached.

\subsection{Golden Section Search (GSS)}

GSS is named after the function values of three testing points, whose distances form a golden ratio. As shown in Fig. 4, a line starting from $X_{l}$ and ending at $X_{u}$ can be divided into two line segments by the intermediate points $X_{l}$. The ratio of the whole line to the larger segment is equal to the ratio of the larger segment to the smaller segment. The relationship can be described in a mathematical way as:

$$
\frac{l_{1}+l_{2}}{l_{1}}=\frac{l_{1}}{l_{2}}
$$

where $l_{1}$ is the length of the larger segment and $l_{2}$ is the length of the smaller one. If $l_{1} / l_{2}$ is defined as $\varphi$, the Equation (4) can be further express as:

$$
\varphi^{2}-\varphi-1=0
$$


After solving the above equation, the positive root is the golden ratio which is equal to 1.61803398874989. Like bisection search [6], GSS releases another intermediate point $X_{2}$, whose position is also chosen in terms of the golden ratio but in an opposite direction.

By successively comparing the values of intermediate points and replacing the upper or lower bound with the internal point, the searching range can be gradually narrowed and the MPP is obtained as the midpoint of a small interval at last. As cleverly placing the intermediate points, recalculation of the integrated module is not required and thus the algorithm speed is improved. However, GSS only works for an unimodal function. When PV modules work under a partial shadow condition, a global search technique is required to assist the tracking process.

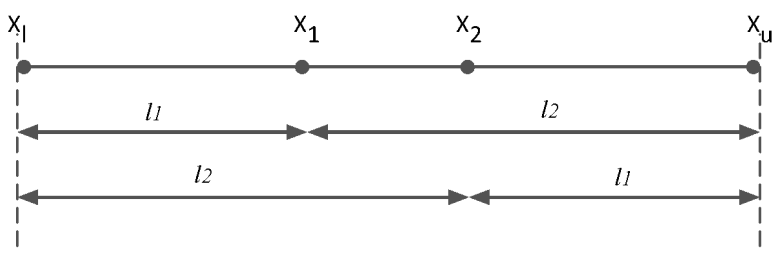

Fig. 4. Intermediate points set of GSS algorithm

\section{$4 \quad$ Experimental Results}

The performance of MPPT algorithms is verified by using computer simulation. The PV system introduced in [12] is selected in this paper. It consists of a PV module

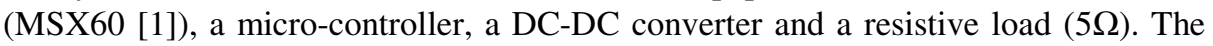
MPPT algorithms are performed by the micro-controller and the computed operating voltage is used to maximize the output power. Fig. 5 shows the testing environment data. As is defined in [8], the feasibility of the MPPT algorithms is evaluated by the MPPT efficiency, which can be expressed as

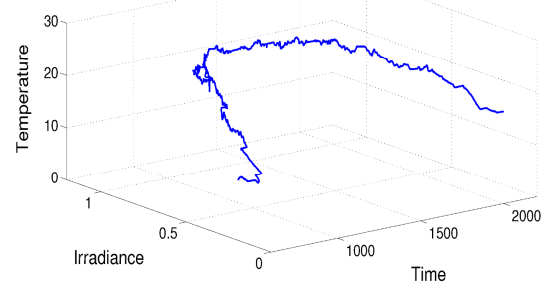

(a)

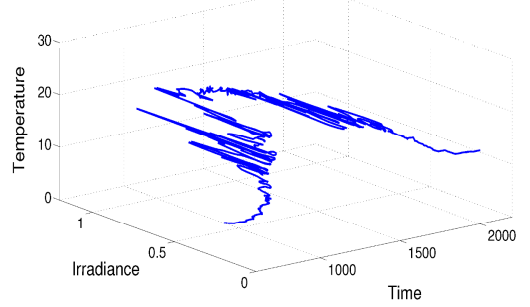

(b)

Fig. 5. Testing environments (a) a sunny day and (b) a cloudy day 


$$
\eta_{M P P T}=\frac{\int P_{\text {actual }}(t) d t}{\int P_{\max }(t) d t} 100 \%
$$

where $P_{\text {actual }}$ and $P_{\max }$ are the simulated PV power and the theoretical maximum $\mathrm{PV}$ power respectively.

Table 1 compares the performance of MPPT algorithms. By comparing the incremental and instantaneous conductance of the PV array, IncCond algorithm gains slightly higher accuracy than $\mathrm{P} \& \mathrm{O}$ method. However, the accuracy of the two direct search methods are reduced about $10 \%$ as the input data of a cloudy day was chosen. GSS shows high speed and MPPT efficiency both in a cloudy day and a sunny day.

Table 1. Performance comparison of MPPT algorithms

\begin{tabular}{|c|c|c|c|c|}
\hline Algorithms & Parameters & Environment & $\begin{array}{c}\text { MPPT } \\
\text { efficiency }\end{array}$ & $\begin{array}{c}\text { Elapsed } \\
\text { fictime }\end{array}$ \\
\hline \multirow{2}{*}{ P\&O } & \multirow{2}{*}{ Increment $=0.01 \mathrm{~V}$} & Sunny day & $99.9699 \%$ & $5.697698 \mathrm{~s}$ \\
\cline { 3 - 5 } IncCond & Increment $=0.01 \mathrm{~V}$ & Sunny day & $99.9722 \%$ & $0.980223 \mathrm{~s}$ \\
\cline { 3 - 5 } & Absolute error=0.01 V & Cloudy day & $90.7850 \%$ & $0.999242 \mathrm{~s}$ \\
\cline { 3 - 5 } GSS & Sunny day & $99.9999 \%$ & $25.8170 \mathrm{~s}$ \\
\hline \multirow{2}{*}{ PSO } & $\begin{array}{c}\text { Absolute error }=0.01 \mathrm{~V} . \\
\text { Population size }=15, \\
\text { Inertia weight }=0.5\end{array}$ & Sunny day & $99.9937 \%$ & $105.1850 \mathrm{~s}$ \\
\cline { 3 - 5 } & \multirow{2}{*}{\begin{tabular}{c} 
Cloudy day \\
\cline { 3 - 5 }
\end{tabular}} & & $99.9773 \%$ & $109.5920 \mathrm{~s}$ \\
\hline
\end{tabular}

\section{Discussion and Conclusion}

Several direct search methods and module-integrated MPPT algorithms are presented in this paper. Also, the effectiveness and efficiency of these algorithms are analyzed. Algorithm complexity, tracking speed, robustness and range of effectiveness are all the factors affecting PV system efficiency. With the ease of implementation, acceptable MPPT efficiency and low cost sensors, the direct search algorithms have been widely used for a long time. Many improvements have been proposed for direct approaches to obtain a better performance. Nevertheless, the inflexible searching method becomes the bottleneck of further development. High-performance low-cost IC technologies and accurate modeling manners have recently widen the development space for module-integrated methods. These MPPT algorithms not only improve the MPPT efficiency, but also enhance the processing capability for rigorous work environment (e.g. partial shadow conditions).

Based on the accurate sampling data, many new design manners can be considered. One of the most known disadvantages of module-integrated methods is the complex computation of a module function. This increases the hardware cost and slows down the convergence speed. If the output of a PV module can be directly expressed as a mathematical expression in terms of several environment variables, many negative features of module-integrated algorithms can be eliminated. Furthermore, more practical optimization techniques, such as gradient method, can be 
applied in a multivariable controlled system. The specially designed PV system will be much cheaper and faster than the computational intelligence algorithm, whereby tracking MPP is based on a large number of random points.

\section{References}

1. 60-Watt Multicrystalline Photovoltaic Module ovoltaic Module Datasheet. BP (2002)

2. Al-Amoudi, A., Zhang, L.: Optimal control of a grid-connected pv system for maximum power point tracking and unity power factor. In: Seventh International Conference on Power Electronics and Variable Speed Drives (Conf. Publ. No. 456), pp. 80-85 (September 1998)

3. Castaner, L., Silvestre, S.: Modelling Photovoltaic System using PSpice. John Wiley \& Sons Inc. (2002)

4. Chen, L.R., Tsai, C.H., Lin, Y.L., Lai, Y.S.: A biological swarm chasing algorithm for tracking the pv maximum power point. IEEE Transactions on Energy Conversion 25(2), 484-493 (2010)

5. Deb, K., Pratap, A., Agarwal, S., Meyarivan, T.: A fast and elitist multiobjective genetic algorithm: Nsga-ii. IEEE Transactions on Evolutionary Computation 6(2), 182-197 (2002)

6. Foster, I., Kesselman, C.: Practical Optimization: Algorithms and Engineering Applications. Springer (2007)

7. Hua, C., Lin, J., Shen, C.: Implementation of a dsp-controlled photovoltaic system with peak power tracking. IEEE Transactions on Industrial Electronics 45(1), 99-107 (1998)

8. Hussein, K.H., Muta, I., Hoshino, T., Osakada, M.: Maximum photovoltaic power tracking: an algorithm for rapidly changing atmospheric conditions. IEE Proceedings Generation, Transmission and Distribution 142(1), 59-64 (1995)

9. Ishaque, K., Salam, Z., Syafaruddin: A comprehensive matlab simulink pv system simulator with partial shading capability based on two-diode model. Solar Energy 85(9), 22172227 (2011)

10. Kennedy, J., Eberhart, R.: Particle swarm optimization. In: Proceedings of IEEE International Conference on Neural Networks, vol.4, pp. 1942-1948 (November/December 1995)

11. Nguyen, D., Lehman, B.: An adaptive solar photovoltaic array using model-based reconfiguration algorithm. IEEE Transactions on Industrial Electronics 55(7), 2644-2654 (2008)

12. Oi, A.: Design and Simulation of Photovoltaic water pumping system. California Polytechnic State University (2009)

13. Salameh, Z., Taylor, D.: Step-up maximum power point tracker for photovoltaic arrays. Solar Energy 44(1), 57-61 (1990)

14. Salas, V., Olas, E., Barrado, A., Lzaro, A.: Review of the maximum power point tracking algorithms for stand-alone photovoltaic systems. Solar Energy Materials and Solar Cells 90(11), 1555-1578 (2006)

15. Shao, R., Chang, L.: A new maximum power point tracking method for photovoltaic arrays using golden section search algorithm. In: Canadian Conference on Electrical and Computer Engineering, CCECE 2008, pp. 619-622 (May 2008) 